\title{
Italo Calvino y un caso de inconmensurabilidad Entender a Kuhn mediante un cuento
}

\author{
Rubén Padlubne ${ }^{1}$
}

\section{Introducción}

Desde los tiempos de la Grecia clásica se utilizaron formas ficcionales para hacer y enseñar filosofía. Así, por ejemplo, una y otra vez nos remitimos al mito de la caverna. Más allá de la ubicación de fronteras entre ambos universos conceptual- discursivos, resulta claro que la literatura es una herramienta fértil para pensar problemas filosóficos porque los textos con estatuto ficcional suponen teorías. Estos textos muestran el doble carácter de la ficción: lo empírico y lo imaginario, y, si son importantes, se sumergen en la turbulencia de lo real desdeñando la actitud ingenua.

En esta dirección, y como parte de un desarrollo de más de doce años de investigación en Didáctica de la Epistemología, ${ }^{2}$ en este artículo propongo tres cuestiones: primero, exponer nuestra propuesta didáctica para enseñar un contenido perteneciente a un curso de Introducción al Pensamiento Científico, en el Ciclo Básico Común de la UBA, utilizando el cuento "Los dinosaurios" de Italo Calvino; segundo, justificar la posibilidad del contraste filosofía de la ciencia- literatura- ciencia; y en tercer lugar, dar cuenta de los vínculos entre las nociones de inconmensurabilidad, relevantes en la obra de Thomas Kuhn, y el mencionado relato de Calvino.

\section{Propuesta didáctica}

Nuestra propuesta didáctica consiste en un análisis contrastivo entre un texto ficcional y algunas nociones de la teoría del cambio científico planteadas por Kuhn para que los estudiantes comprendan el concepto de inconmensurabilidad como imposibilidad de traducción (parcial o total) entre taxonomías léxico- conceptuales diferentes y, a la vez, comprendan la necesidad de interpretación para entender teorías de otros paradig-

\footnotetext{
1 Rubén Padlubne UBA/UNTREF/UTN/UNGS/ISP J.V. González

Este artículo fue publicado en el Cuaderno de Trabajo Número 1 Cuaderno de trabajo - 2da. Época Año 1, № 1 ISSN 1667 - 4995 abril de 2012, Hemos publicado, en nuestro Dossier, la mayoría de los Cuadernos de Trabajo que preceden a su publicación digital. Este artículo, como otros que se irán publicando, corresponde a material que no salió a luz en forma virtual.

2 Se trata de sucesivos proyectos desde 1995 dirigidos primero por el Dr Ricardo Guibourg y luego por la Dra Cristina Ambrosini, los que posibilitaron- entre otras tantas publicaciones- la publicación del libro El Pensamiento Científico y el Método Educativo, Buenos Aires, Editorial Educando, 1999
} 
mas. Lo fundamental de esta propuesta es que la comprensión de la filosofía del mencionado autor se lleve a cabo en un proceso que involucra operaciones cognitivas similares a las empleadas por Kuhn: razonar analógicamente, identificar tipos de vínculo entre afirmaciones con distintos grado de generalidad, efectuar una lectura interpretativa, y dar cuenta de cortes epistemológicos.

Para alcanzar este propósito diseñamos un instrumento orientador para los alumnos, restrictivo y dispersivo a la vez, que consiste en una serie de consignas. La actividad solicita responder en forma escrita los interrogantes del instrumento didáctico y luego generar un intercambio oral que cumple varias funciones: ajustar y reformular la comprensión previa, profundizar el conocimiento de los contenidos comprometidos, y convocar a la participación de los alumnos como actores reales en una situación argumentativa compleja para ellos.

Una parte del mencionado instrumento didáctico, incluidas las consignas, es la siguiente:

1) Lea con especial atención las nociones de paradigma e inconmensurabilidad. Extráigalas.

2) Sin proponerse responder nada, lea completamente el cuento "Los dinosaurios", de Italo Calvino.

3) Lea nuevamente el cuento para responder las consignas siguientes:

a- el narrador- protagonista, Ofwfq, ¿vive en una época o vive en varias? Justifique con fragmentos del cuento.

b- escriba una lista con todos los términos con que los otros personajes nombran a Ofwfq (la extensión).

c- ¿qué sucede con el reconocimiento entre Ofwfq y los "Nuevos"? ¿La extensión enumerada se vincula con el problema del reconocimiento?

d- ¿por qué el hijo de Ofwfq no ve el parecido que tiene con su padre en el final del relato?

e- el término "dinosaurio" expresa: e1: un universal

e2: algo particular

f- escriba asociaciones posibles entre las nociones de paradigma e inconmensurabilidad y el relato leído.

4) Teniendo en cuenta las nociones de conocimiento acumulativo y conocimiento discontinuo, las tres versiones de la inconmensurabilidad, y el concepto de interpretación, opte y justifique su elección: en el texto de Calvino...

a- se pone de manifiesto la cuestión de

a1- la traducción

a2- la interpretación

a3- ninguna de ambas operaciones

b- ¿hay dificultad y/o imposibilidad de comparar? SI- NO

c- se manifiesta así una o más de las versiones de la inconmensurabilidad?: SI- NO. Justifique.

d- Calvino propone un conocimiento acumulativo o discontinuo (opte por una) 
e-Tomando en cuenta lo que usted conoce de la teoría de Kuhn, el personaje se llama Ofwfq porque

1- no es nombre de un personaje sino el "ejemplar exitoso" tomado como modelo durante la vigencia de un paradigma.

2- es una palabra deliberadamente impronunciable para expresar, implícitamente, el fracaso de lo universal para todo tiempo y lugar.

3- es el nombre particular de una cultura, es decir algo parecido a un paradigma.

4- es el nombre del científico o filósofo que toma distancia con las épocas, los paradigmas y las distintas culturas, porque tiene una mirada, un método o una razón universal.

\section{Juguetes rabiosos: filosofía y literatura}

La pertinencia de contrastar filosofía de la ciencia y literatura se explica mediante varios tópicos. Uno de ellos es que la versión del cambio científico propuesta por Kuhn se aproxima a la concepción canónica de los cambios en literatura: amplios períodos orientados cada uno de ellos por una sensibilidad singular y convencional que se suceden separados por abruptos cambios de convención (la novela burguesa desplazó las formas épicas predominantes durante varios siglos). Por otra parte, y más allá de las obvias diferencias entre ambos discursos (filosófico y literario), algunos teóricos (L.Geymonat, H.Putnam, E.Mari) creen que comparten una común sustancia espiritual: preguntan por lo "real" sin la determinación de lo inmediato ni del dato, y tienen una voluntad de saber que incluye los mundos posibles. Son modos de representación y una de las maneras más habituales de representación científica es el modelo, comparable con la metáfora literaria (también utilizada en ciencia). El modelo y la metáfora actúan analógicamente, razonamiento privilegiado por Kuhn al explicar cómo funciona la "ciencia normal" en común su carácter "universal". Las verdades importantes de la ficción se ejemplifican mediante casos específicos y convincentes; en tanto que la segunda se manifiesta en términos abstractos. Así, mientras que la literatura expresa una suerte del "universal concreto hegeliano", la ciencia se expresa en el "universal abstracto". En este sentido, las leyes científicas, por ejemplo las leyes del gas ideal, siempre representan como irían las cosas en un mundo en el que a determinadas condiciones iniciales le sucederían determinadas consecuencias; pero no se debería esperar que la realidad caótica siga literalmente a las leyes ideales de la ciencia como tampoco se espera que tal "realidad" siga los criterios de la novela realista.

Esta comparabilidad, que se constituye sobre el supuesto de diferencias básicas obvias, también se pone de manifiesto cuando se pondera, desde una perspectiva filosófica, los méritos en función de ciertos fines. Así, por ejemplo, la novela El juguete rabioso, de Roberto Arlt, sintetiza el drama general de la adolescencia simplemente porque representa tal drama mejor que otras ficciones. De modo parecido, Kuhn sostiene que:

3 Las teorías se proponen por parecido con el modelo ejemplar o matriz disciplinar en las distintas versiones que Kuhn expone de su propia Epistemología. 
[...] para ser aceptada como paradigma una teoría debe parecer mejor que sus competidoras; pero no necesita explicar y, en efecto, nunca lo hace, todos los hechos que se pueden confrontar con ella. (Khun, 1993, p.44)

Una ficción lograda y una teoría científica son hipótesis sobre cómo funciona alguna porción del mundo, mostrando así algo de invento similar al juego: constituyen determinados juegos en los que se expresa la pasión por apropiarse de lo real que siempre parece correrse. En esa pasión por "el juego" se hacen juguetes rabiosos en tanto las ficciones literarias y las teorías se constituyen en una relación tensional con aquello que representan, en un caso; y con lo que explican y predicen, en el de la ciencia. Así, "rabioso" es una metáfora sobre el problemático y complejo vínculo que establecen con lo "real".

El giro lingüístico y la pragmática de Austin aproximan filosofía y literatura en tanto ambas empiezan a ser analizadas semióticamente, es decir, como sistemas de signos, esto es, modos de producción de sentido, situando lo sustancial del análisis del lenguaje en la dimensión pragmática. No en el lenguaje, sino en su análisis de la historia de la ciencia, Kuhn también es un pragmático pues los supuestos compartidos por una comunidad, nos dice, resultan de una teoría exitosa. ¿Pero acaso una obra literaria tiene alguna utilidad? Sin duda. Por ejemplo, leer en ella anticipaciones de hipótesis y teorías de lo más diversas. En esta dirección, el recorrido curvilíneo trazado en los cielos por el Dante en la Divina Comedia adelanta el distanciamiento con el espacio plano de Euclides a favor de un espacio más curvo que plano que comienza a proponerse en el S.XVII. Shakespeare, con Coriolano, anticipa la teoría de la lucha de clases de Marx. A mediados del siglo XVII, Cyrano de Bergerac publicó un texto ficcional, Viaje a la Luna, donde se presentan siete formas diferentes de efectuar tal viaje; pero lo sorprendente es que aproximadamente cuarenta años antes que Newton plantease su teoría científica, uno de los viajes diseñados por Cyrano es mediante cohetes que vencen al aire que se resiste, es decir, aquello que luego Newton denominaría y explicaría como gravedad. La experiencia estética es un modo de conocer el mundo, tanto si se la considera como significación producida por relación entre signos, es decir semiológica, como por su valor referencial. La mirada semiológica no pretende negar la función referencial, sino quitar a la referencia su dudosa probada autoridad en el proceso de significación (En este sentido, el hipotético deductivo sofisticado pulverizó la certeza en el hecho).

La conciencia del lenguaje potencia nuestra capacidad de análisis. En este sentido tal vez debamos admitir, una vez más, que la diferencia entre filosofía y literatura radica en el modo de usar las palabras, es decir, en distintos actos de habla y en diferentes retóricas. En cuanto a lo segundo, la filosofía tiene un lenguaje explicativo y descriptivo (hay excepciones), en cambio lo literario nace de un gesto de extrańamiento, como sostienen los formalistas rusos. Por otra parte, Gadamer (1996) afirma que ambas comparten la propiedad de no ser falsables ni falsas, pues no hay una regla exterior que pueda juzgarlas.

\section{Las tres versiones de la inconmensurabilidad}

Kuhn (1993) efectuó un desarrollo con sucesivas reformulaciones de su teoría en el que se separó y criticó la noción del progreso como acumulación continua de conoci- 
miento propuesta por el positivismo. Esta creencia se debe a la manera de concebir la ciencia mediante un enfoque "presentista", es decir, como un conjunto de escalones que conducen a un estado actual. Kuhn propone un modelo no presentista, en el que se utilizan técnicas interpretativas de otra cultura para analizar textos científicos de otras épocas (Kuhn, 1989). Así, es posible interpretar teorías lejanas en el tiempo pero no siempre podemos hacer comparaciones entre teorías. A su vez, al sostener que no hay un procedimiento sistemático, universal y neutral para decidir la elección entre paradigmas y/o teorías rivales se rechaza la evolución de la ciencia como un proceso acumulativo, esto es, progresivo cuando hay cambio de paradigma ${ }^{4}$, de lo que según Kuhn se desprende que entre sucesivas teorías (y/o paradigmas) rivales hay inconmensurabilidad. Así, la evaluación de las teorías alternativas se vuelve una cuestión mucho más complicada que una simple comparación debido al cambio conceptual entre aquellas.

La noción de inconmensurabilidad desató una fuerte controversia por sus implicancias filosóficas de amplio alcance y renovó la discusión sobre la racionalidad científica. Kuhn ofrece tres versiones diferentes de inconmensurabilidad. La primera aparece en $\mathrm{La}$ estructura de las revoluciones cientificas donde presenta una formulación global: se trata de una relación entre paradigmas sucesivos. La segunda versión (inconmensurabilidad local), en los años setenta, restringe el dominio de la relación de inconmensurabilidad: se trata del vínculo que se manifiesta entre teorías incluidas en paradigmas sucesivos $y$, más precisamente, sus léxicos:

[...] dos teorías son inconmensurables cuando no hay ningún lenguaje neutral, o de cualquier tipo, al que ambas teorías puedan traducirse sin resto o pérdida. (Khun, Th. 1989, p.99)

Es decir, cuando no son completamente traducibles entre sí. Por su parte, la tercera versión de inconmensurabilidad, expuesta a partir de los años ochenta, está centrada en la cuestión ontológica que se sintetiza en el problema del "cambio de mundos", es decir en el plano del esquema conceptual. Si en la segunda versión el centro era la cuestión de la traducibilidad entre palabras, en la tercera versión se plantea que las diferencias entre "los mundos" constituidos por distintas teorías que se ocupan de referentes similares son de carácter conceptual.

\section{Un dinosaurio bajo la lupa: versión literaria de Kuhn}

\subsection{Discontinuidad y desacuerdo}

El cuento de Calvino narra la vida de un dinosaurio, Ofwfq, que vive aún después del Cretáceo cuando ya habrían desaparecido sus congéneres. El narrador del cuento es el mismo Ofwfq, quien hace las veces de un historiador que atraviesa el tiempo llegando al presente. Deambulando, este narrador- protagonista se encuentra con descendientes de su especie: "los Nuevos", quienes no lo reconocen como dinosaurio, aunque a la vez dudan respecto de su pertenencia y le atribuyen distintas características de los temidos megaterios. Así, aparece una mulata, mezcla de dinosaurio y Nuevo, que seduce a Ofwfq y de tal

4 Bien sabemos que durante la "ciencia normal" sí postula un proceso acumulativo. 
encuentro nace un pequeño muy parecido al protagonista. La historia termina cuando el padre dinosaurio y su hijo se preguntan mutuamente "Quién eres tú". Asombrado, Ofwfq, responde "Nadie"; en tanto que el pequeño dice con certeza: "lo saben todos, soy un Nuevo". Por lo dicho, es clara la centralidad del problema del reconocimiento.

Todo el relato "Los dinosaurios" puede ser presentado como expresión de la diferencia entre la concepción de desarrollo científico acumulativo continuo guiado por un universal, y la perspectiva kuhniana de carácter historicista discontinuo. Tal contraste se materializa en el texto de Calvino mediante la tensión entre una noción universal de "dinosaurio" y los sentidos particulares que se le atribuyen.

En la misma dirección, "Los dinosaurios" puede ser pensado como una mostración del "desacuerdo racional" entre distintas perspectivas, es decir como un auténtico ejercicio de argumentación y deliberación y no una mera secuencia de diálogos y comentarios. En este sentido, vale recordar que Kuhn concibe el desacuerdo como parte necesaria de la producción científica. Así como la elección de teorías está fincada en compromisos pragmáticos, ontológicos y metodológicos compartidos por una comunidad durante un período histórico, análogamente, en el cuento de Calvino la percepción y conceptualización del término "dinosaurio" es relativa a cada época. Este relativismo se manifiesta en Calvino en la extensión del término "dinosaurio". Precisamente, esta extensión es fundamental en el hilo argumentativo del texto en tanto ella materializa una serie de diversos significados.

Tal diversidad de significados en el universo ficcional es "equivalente" a la divergencia de juicios permitida por el carácter flexible de los valores epistémicos en la teoría kuhniana y, a la vez, un ejemplo de la distribución de riesgos en las coyunturas de elección en una comunidad científica.

\subsection{Extensión, reconocimiento, interpretación}

La mencionada extensión del término "dinosaurio", el protagonista, incluye "monstruo", el "más fuerte", el "feo", "personaje ridículo", "cómico", "el más triste”; pero, sobre todo es "un extranjero"; designaciones proferidas por "los Nuevos", animales descendientes del dinosaurio. Estos modos de nombrar están en tensión con la univocidad del término "dinosaurio" expresando así las distintas esquematizaciones del mundo en el soporte del lenguaje. Tales esquemas mentales posibilitan una comprensión sinóptica del mundo, en tanto matrices cognitivas que llevan a los enunciadores a seleccionar un aspecto del narrador- protagonista (jerarquizándolo así por encima de otros): para unos es cómico, para otros, triste. Sin embargo, ya hemos señalado que lo más relevante es su extranjería, es el otro, lo más alejado al reconocimiento, el significado difícil o imposible de traducir.

El problema del reconocimiento, sostiene Kuhn, polemizando con Putnam y con Quine, es el de la diferenciación entre interpretación y traducción. Así, en su trabajo ¿Qué son las revoluciones científicas? afirma que:

[...] la traducción es algo efectuado por quien sabe dos idiomas. Al enfrentarse con un texto en uno de estos idiomas el traductor sustituye sis- 
temáticamente palabras o secuencias de palabras de un idioma por las del otro idioma, a fin de producir un texto equivalente. (Khun, 1989, p.103)

En cambio, en la interpretación puede suceder que quien:

Efectúa la interpretación domine inicialmente sólo una lengua [...] Al principio, el trabajo puede consistir, total o parcialmente, en ruidos o inscripciones ininteligibles [...] quien interpreta busca un sentido, se esfuerza por inventar hipótesis que hagan inteligible la inscripción. Si tiene éxito, lo que él ha hecho en primera instancia es aprender una nueva lengua. (Khun, 1989, pp.104-105)

Por ejemplo, los términos "fuerza" y "masa", sostiene Kuhn, funcionan diversamente en distintas teorías (las de Newton y Einstein), de manera que el aprehender los significados de la otra teoría es interpretar. En esta dirección, los Nuevos, personajes del cuento de Calvino, han aprendido ciertos rasgos sobre los dinosaurios, pero, situados en otro tiempo y otra cultura (entonces, otro paradigma) la noción anterior de dinosaurio se les aparece como un ruido pues no pueden traducirla. En este sentido, en el relato hay un episodio significativo en cuanto parece ilustrar la noción de inconmensurabilidad, es decir, imposibilidad de "traducción" o reconocimiento: los Nuevos y Ofwfq encuentran un esqueleto de dinosaurio, lo miran pero solamente Ofwfq sabe que es un dinosaurio como él, quien afirma:

Aquellos huesos, aquellos colmillos, aquellos miembros exterminadores hablaban una lengua ahora ilegible, ya no decían nada a nadie, salvo aquel vago nombre que había perdido relación con las experiencias del presente. (Calvino, 1991, p.133)

Con el dinosaurio sucede algo distinto. En tanto protagonista, a veces vacila en su reconocimiento de lo que sucede con los otros personajes, pero dado que a la vez es el narrador, un especial narrador que- según ya expusimos- asume el carácter de historiador pues comprende y da cuenta del paso del tiempo al estar en distintas épocas (desde el Triásico y el Jurásico hasta los tiempos contemporáneos).

\subsection{El cambio}

La narración de Calvino presenta recurrentemente sintagmas referidos al cambio: "El mundo ha cambiado: no reconocía ni los montes ni el río ni las plantas"(Calvino, 1991, p.120), dice desconcertado el protagonista- historiador, pues confiesa que ha vivido más de cincuenta millones de años y en la última línea del relato nos enteramos que ha llegado a los tiempos contemporáneos y se confunde con la multitud. El desconcierto de los Nuevos y las dificultades del dinosaurio- historiador para convivir con aquellos pone de manifiesto el cambio de percepción y de conceptualización que conlleva el paso del tiempo. Como afirma Kuhn, desde paradigmas diferentes parece que estamos en "mundos diferentes".

Hay un episodio en el que nuestro protagonista intenta abandonar al grupo de animales porque se escuchan fuertes ruidos, entonces lo apostrofan riéndose: “¿Por qué 
escapas, pareciera que hubieras visto un dinosaurio?” Sin embargo, Ofwfq nos comunica que se trata de una risa forzada, poniendo así de manifiesto la duda, la posibilidad de alcanzar el mismo significado que Ofwfq ya conoce; así, en el diálogo muestra, a través de la dificultad o imposibilidad de reconocimiento, el cambio de percepción y conceptualización, es decir, aquello que se puede asociar con la cuestión de las taxonomías compartidas por distintas teorías pertenecientes a paradigmas sucesivos. Por este camino, en un momento de la narración el dinosaurio dice que:

[...] las historias que de nosotros contaban los Nuevos estaban tan lejos de mi experiencia que hubieran debido dejarme indiferente, como si hablaran de extraños. Y sin embargo, escuchándolas yo comprendía que nunca había pensado en lo que parecíamos a los demás. (Calvino, 1991, p.123)

\subsection{La referencia, el cuerpo}

En la Estructura de las Revoluciones Cientificas leemos que una teoría debe rechazar o desplazar a la anterior para que pueda ser asimilada por la comunidad, afirmación ejemplificada con la aceptación de la teoría de la conservación de la energía que exigió el rechazo de la teoría calórica.

¿Qué elemento desplaza a otro elemento en Calvino? Para responder debemos recordar el uso metafórico del término "dinosaurio", que en la cultura contemporánea significa dos cosas diferentes y opuestas: fuerza y vejez. Los Nuevos quieren, necesitan, ser los fuertes de su época y, para ello, necesitan desplazar al dinosaurio, dejándole solo el sentido de "lo viejo".

A lo largo del relato hay una lucha entre reconocimiento y no reconocimiento, pero, dice el narrador, que para los Nuevos los huesos de dinosaurio ya "no hablan una lengua". El reconocimiento es interpretación, y esta finalmente fracasa al imponerse el significado propio del período. El cuerpo del dinosaurio es un cuerpo que ha cambiado de significado, pues tanto para Calvino como para Kuhn no hay nada exclusivamente cuerpo, todo es indisolublemente conceptual- empírico. La imposición de "lo nuevo" se afirma en el episodio con el que se cierra la narración, muy importante porque es la conclusión argumentativa. En tal episodio Ofwfq se enfrenta con su hijo, quien le dice rotundamente que es "un Nuevo"; en cambio, el padre dice que es "Nadie". "Nadie" es un cuerpo y un nombre ininteligibles; precisamente Ofwfq que vivió en todos los tiempos, la "noción universal", se configura como significante vacío para el pequeño descendiente de dinosaurio, quien es ignorante de lo que el nombre Dinosaurio significa. Es "nadie" porque no puede ser percibido ni comprendido en tanto cumple la función intemporal del universal; así Calvino concluye como Kuhn que el universal es inviable.

\section{Conclusión}

El contraste entre la teoría de Kuhn y el cuento de Calvino tiene el propósito de contribuir en el desarrollo de la competencia argumentativa de los estudiantes, situándolos frente a un problema poco habitual. Este tipo de actividad argumentativa- deliberativa constituye una experiencia que permite más que recuperar ciertos contenidos: convoca 
a un ejercicio de lectura compleja en el que se imbrican lo imaginativo y lo sistemático favoreciendo otra manera de pensar lo "real".

Por otra parte, al tratarse de un diseño de experiencia con estudiantes que acaban de iniciar sus estudios universitarios, el desafío de asociar contenidos de una introducción a la Epistemología con la ficción literaria los pone en la situación de reflexionar sobre la noción de "mundos posibles", situación doblemente significativa en tanto la ciencia también utiliza tal noción, y ellos atraviesan un período de sus vidas en el que deben elegir precisamente entre "mundos posibles" para sus propias vidas.

Por otra parte, la teoría de Kuhn plantea un doble tipo de desarrollo para la ciencia, el acuerdo (la ciencia normal) y el desacuerdo (la revolución). Los acuerdos y desacuerdos entre los personajes en el relato de Calvino favorecen el intercambio respecto del valor de tales modos de la racionalidad y una reflexión metacognitiva sobre nuestros diversos modos de capturar lo "real".

\section{Bibliografía}

- Calvino, I. (1991). "Los dinosaurios", Las cosmicómicas, Barcelona, Minotauro.

- Díez, J. A. y Moulines, C.U.; (1997) Fundamentos de Filosofía de la Ciencia, Barcelona, Ariel.

- Gadamer, H. (1996). Estética y Hermnéutica, Madrid, Tecnos.

— Kuhn, Th. (1993). La Estructura de las revoluciones cientificas, México, Fondo de Cultura Económica.

— Kuhn, Th. (1989). ¿Qué son las revoluciones científicas? Barcelona, Paidós.

- Lorenzano, C. (1998). La estructura del conocimiento cientifico, Buenos Aires, Zavalía.

— Lynch, E. (2007). Filosofía y/o literatura, Buenos Aires, Fondo de Cultura Económica, 2007.

— Marí, E. (1999). "Ficción literaria y científica” , (sin otros datos).

— Putnam, H. (1994)Las mil caras del realismo, Barcelona, Paidós. 